\title{
Air cycle combined heating and cooling for the food industry
}

\author{
A.M. Foster ${ }^{1}$, T. Brown ${ }^{1}$, A.J. Gigiel ${ }^{2}$, A. Alford ${ }^{3}$ and J.A. Evans ${ }^{1}$ \\ ${ }^{1}$ London South Bank University (LSBU), Churchill Building, Langford, North Somerset, \\ $B S 405 D U, U K$. \\ Tel: +44 (0)117 9289239, Fax: +44 (0)117 9289314,Email:alan.foster@lsbu.ac.uk \\ ${ }^{2}$ CCC Consultants, Fairview, Moorlynch, Bridgwater, Somerset, TA7 9BY, UK \\ ${ }^{3}$ Corac Group plc., Brunel Science Park, Kingston Lane, Uxbridge. UB8 3PQ, UK
}

\begin{abstract}
In the food industry there is often a need to both cook and cool food. Heating and cooling processes are rarely directly linked due to heat from refrigeration processes being insufficient to cook food. Therefore cooling and cooking is usually provided by separate pieces of equipment. This paper presents an air cycle system where the hot air was used for heating and the cold air used for fast freezing.
\end{abstract}

The apparatus used a bootstrap unit developed for aircraft air conditioning which was unable to run at the low temperature required for food freezing, so a parallel compressor was added to enable the system to operate at the low temperature required for food freezing. This approach allowed temperatures as low as $-140^{\circ} \mathrm{C}$ and as high as $234^{\circ} \mathrm{C}$. The system cooked and froze beef burgers, and at the same time heated water up to $98^{\circ} \mathrm{C}$. 
Keywords: Brayton, Cooking, Cooling, Centrifugal compressor, Food, COP

Brayton, Cuisson, refroidissement, Compresseur centrifuge, Produit alimentaire, COP 


\section{NOMENCLATURE}

$c_{\mathrm{p}} \quad$ specific heat at constant pressure $\left(\mathrm{J} \cdot \mathrm{kg}^{-1} \cdot \mathrm{K}^{-1}\right)$

m mass $(\mathrm{kg})$

$\mathrm{N} \quad$ Rotational speed $\left(\mathrm{s}^{-1}\right)$

$\dot{m} \quad$ mass flow rate $\left(\mathrm{kg} . \mathrm{s}^{-1}\right)$

P Total electrical power of compressors (W)

Q $\quad \operatorname{load}(\mathrm{W})$

r pressure ratio

$\mathrm{T}$ temperature $(\mathrm{K})$

$\mathrm{V} \quad$ aerodynamic speed $\left(\mathrm{s}^{-1} \cdot \mathrm{K}^{-0.5}\right)$

W work (J)

$\Delta \mathrm{T} \quad$ Temperature difference across load (K)

$\gamma \quad$ ratio of specific heats, $\mathrm{c}_{\mathrm{p}} / \mathrm{c}_{\mathrm{v}}$

$\eta \quad$ efficiency

subscripts

$\mathrm{T}$ turbine, turbine inlet

cool cooling

cor corrected

c compressor, compressor inlet

heat heating

ht heater tape

i inlet

is isentropic

o outlet 


\section{INTRODUCTION}

Large quantities (US\$3.2 trillion) of food are processed each year by the worldwide food industry (Regmi and Gehlhar, 2005). Much of this processed food is heated and then frozen during production.

Food is predominantly frozen in air-based blast coolers operated using a direct expansion refrigeration system. Most direct expansion systems are single or two stage compressor systems but are constrained to operate above approximately $-40^{\circ} \mathrm{C}$.

Occasionally if low temperatures are required a cascade system may be employed. Cascade vapour compressions systems are complex to install, have a low COP and are therefore expensive to operate.

Alternatively if fast freezing at low temperatures is required, food manufacturers may use liquid nitrogen (LIN) systems. LIN systems are total loss refrigeration systems (refrigerant evaporates and is vented into the atmosphere) and require LIN to be supplied and stored at the food factory. LIN systems are relatively cheap to install but are expensive to operate. Most food is cooked in dry air, steam or water at temperatures above $70^{\circ} \mathrm{C}$. Potentially heating and cooling can be carried out simultaneously. By utilising heating as a by-product of the cooling process this can increase the overall process efficiency. The temperature at which heat is rejected from the refrigeration cycle is the critical factor that defines how useful the heat can be for cooking. In most well designed direct expansion refrigeration systems the heat rejected from the system is not high enough to be economically useful. One exception to this is in cold stores where the compressor discharge 
gas is commonly used to heat pumped glycol under floor heaters to prevent the ground under the cold store freezing and damaging the store floor. Another is the relatively low grade heat that can be reclaimed from the oil coolers of screw compressors where a significant amount of the compressor motor power can be absorbed in the oil. Systems have been developed that use heat from the compressor discharge or compressor oil coolers to pre-heat water in a boiler. Although these were traditionally considered uneconomic, with improved building insulation, the low grade heat available becomes more attractive. In an example presented by Das (2000), a combined heating and cooling system that provided under floor heating and pre boiler water heating to $35^{\circ} \mathrm{C}$ gave a pay back period of 2.5 years.

For larger systems, heating and cooling using $\mathrm{CO}_{2}$ have been developed. Although $\mathrm{CO}_{2}$ systems have some limitations in the level of heating that they can provide, they have potential for heating water or for applications where high-temperature heat is not required. Sarkar, Bhattacharyya and Gopal (2006) proposed a system to provide simultaneous cooling at $4^{\circ} \mathrm{C}$ and heating at $73^{\circ} \mathrm{C}$ for dairy plants using a transcritical $\mathrm{CO}_{2}$ cycle.

A system to provide chilled and hot water using a transcritical $\mathrm{CO}_{2}$ system has been proposed by Pearson (2006). The concept proposed would provide high temperature hot water for practically no energy input but the capital cost would be higher than an electric heater or gas fired boiler.

Although systems exist for generating heat at low temperature, no systems are currently available that generate sufficient heat to directly cook foods at a temperature of $200^{\circ} \mathrm{C}$ or above. The exception to this is air cycle refrigeration which can generate large 
quantities of reasonably high grade heat, while at the same time producing air at very low temperatures. Several advantages for air cycles can therefore present themselves where there is a linked need for heating (e.g. cooking) and rapid cooling. The very low temperatures allow rapid cooling and freezing, reducing weight loss, residence time, factory footprint for the process and potentially improving quality.

Air cycle refrigeration is based on the reverse Brayton or reverse Joule cycle. It is a gas rather than a vapour cycle, hence the gas does not change phase during the cycle. The Brayton cycle is less efficient than the Rankine cycle used for vapour compression systems as the working fluid does not receive and reject heat at constant temperature. One of the ways of improving the efficiency of air cycle is to use the work carried out in expansion to further compress the air on the high pressure side, lowering the overall energy input for a given expansion pressure ratio. This can be done by mounting the compressor and turboexpander on a common rigid shaft in an arrangement known as a bootstrap system. A further efficiency improvement is to use a recuperative heat exchanger to pre-cool the air into the turbine with the cold air returning from the cooling apparatus.

Air cycle refrigeration has been investigated with a number of different applications in mind, deep freezing (Hou and Zhang, 2009), air-conditioning and desalination on ships (Hou et al., 2008), supermarket refrigerating and ventilated air conditioning (Elsayed et al., 2006), road transport (Spence et al., 2005).

Air cycle is currently dominant in the air conditioning and cabin pressurisation of modern aircraft (Rogers, 1994), where size, weight, reliability and a plentiful supply of compressed air outweigh energy efficiency concerns. More recently air cycle railway 
carriage air conditioning systems have been developed in the UK by Honeywell Aerospace for Germany's new ICE-3 high-speed train, where the robustness and low maintenance requirements contribute to lower life cycle costs than conventional vapour compression systems.

Air offers other important advantages over other refrigerants. If it leaks it has a zero ozone depletion and global warming potential and is non-toxic and non-flammable. The system is far less susceptible to leakage than vapour compression systems and is therefore more reliable. When leaks from vapour compression plant are taken into account the total equivalent warming impact (TEWI) of air cycle can compare favourably with many vapour compression systems.

This paper presents an air cycle system which operates as a closed cycle. The hot air exchanged from the high pressure side was used for heating and the expanded cold air used for fast freezing. Due to lack of available suitable components for generation of low temperature air a novel solution was developed to allow the principle of air cycle refrigeration to be demonstrated at a low cost.

\section{AIR CYCLE SYSTEM}

\subsection{System design}

A T-s diagram for the air cycle is shown in Figure 1. Open and closed configurations for the cycle were considered. In an open system, the working fluid is passed directly over the product. This can lead to problems with ingress of moisture from the product and ambient air and subsequent icing of the heat exchange surfaces. A closed 
system passes the working fluid through heat exchangers and secondary air is circulated over the product by fans. An impingement freezing tunnel was used for trials and the flow rate from the heat exchanger fans in the tunnel was much larger than the flow rate of the working fluid provided by the primary compressor. As a closed system would have less moisture in the cycle and a higher heat transfer to the food, it was chosen over an open system. A schematic of the equipment is shown in Figure 2.

\subsection{System components}

Pressure was generated from an electrically driven rotary high speed compressor (Corac, UK). This was a pre-production oil-free compressor using an external supply of compressed air to supply the air bearings. The motor was driven from an inverter (Vacon Drives UK Ltd) and its speed controlled via a 4-20 mA signal from a computer.

The bootstrap unit comprised a centrifugal compressor driven by an inward-flow radial turbine. It was designed and developed for the BAe Experimental Aircraft Programme and provided by Honeywell Aerospace. The bootstrap contained no oil and bled some of the air passing through it to supply the air bearings.

To heat and cool food, conveyorised tunnels with impingement plates were used, allowing higher heat transfer coefficients at the product surfaces than a conventional blast system. The impingement tunnels were supplied by Air Products (UK), having been modified from tunnels originally developed for use with LIN as the working fluid. 
A shell and tube heat exchanger (SB4528, EJ Bowman Ltd) was used to heat water to $90^{\circ} \mathrm{C}$. Another shell and tube heat exchanger (SB4529, EJ Bowman Ltd) was used to remove remaining heat from the air before passing through the recuperator.

Temperatures were monitored before and after every component using Type-T thermocouples (calibrated at 0 and $100^{\circ} \mathrm{C}$, accuracy $\pm 0.5^{\circ} \mathrm{C}$ ) placed in copper wells such that the thermocouples were located in the centre of the pipes. Pressures were monitored before and after each compressor and turbine (Model PXM219, Omega Engineering Ltd, UK accuracy $0.25 \%$ ).

To enable high cooking temperatures to be produced at the same time as low freezing temperatures, exchange of heat between the high and low temperature sides of the cycle was necessary. This was done in a recuperative heat exchanger or recuperator. This consisted of 4 cross flow compact plate-fin heat exchangers connected together in a countercurrent arrangement to increase effectiveness. The heat exchangers were built for Germany’s ICE-3 high-speed train and supplied by Honeywell Aerospace (UK).

The system was not completely leak free during operation, and contained a larger mass of air when running than when idle. It therefore required both initial and run-time top-up of air, which was added via an open pipe placed at the lowest pressure point in the system (at the inlet of the primary compressor) To reduce the moisture in this top-up air, a desiccant dehumidifier (MLT800) supplied by Munters (UK) Ltd was used.

The high temperature pipes were lagged with RockLap (Rockwool, UK) insulation and the low temperature pipes were lagged with Armaflex insulation (Armacell UK Ltd). 


\subsection{Parallel compressor}

The bootstrap compressor/expander was designed for aircraft air conditioning. The compressor was designed to receive air between 100 and $200^{\circ} \mathrm{C}$ and the turbine was designed to receive air between 20 and $170^{\circ} \mathrm{C}$. Predicted temperatures off the primary compressor and into the bootstrap compressor were within the design conditions of the bootstrap compressor, but those for the turbine were not.

To achieve the air temperatures required for low temperature freezing, a recuperator was used to reduce temperatures into the turbine to well below its design conditions. The density of air passing through the turbine was therefore much higher than it was designed for. This would cause a volumetric flow mismatch between the compressor and the turbine, leading to a greater than designed mass flow rate through the bootstrap compressor. This would move the conditions for the compressor to a higher than designed flow rate where its efficiency would be extremely low (choked flow). As this would have been unacceptable, various modifications were devised to balance the compressor and turbine.

One solution to this fluid-dynamics problem is to block the turbine nozzles, thus reducing the mass flow rate through the system. However, there is also a thermodynamic issue of the reduced inlet temperature to the turbine. The work produced by the turbine and transmitted to the compressor along the shaft is directly proportional to the turbine inlet temperature as shown in eqn. $1 \quad$ (1. Therefore the pressure ratio of the bootstrap compressor drops below design conditions. 


$$
W_{T}=\eta_{T} m_{T} c_{p} T_{T}\left[1-\left(\frac{1}{r_{T}}\right)^{(\gamma-1) / \gamma}\right]=W_{C}=\frac{m_{C} c_{p} T_{C}\left[\left(r_{C}\right)^{(\gamma-1) / \gamma}-1\right]}{\eta_{c}}
$$

To overcome this problem the air flow after the primary compressor was split, so that only a proportion was fed through the bootstrap compressor, thus reducing $\mathrm{m}_{\mathrm{c}}$. The remainder of the flow was compressed by another compressor operating 'in parallel' with the bootstrap compressor. This solved the thermodynamic problems in the bootstrap, in that the same amount of work was done on a smaller mass flow rate in the bootstrap compressor, therefore keeping the pressure ratio high. It also solved the fluid mechanical problems as there is a reduced mass flow rate through the bootstrap compressor.

The parallel compressor was a rotary positive displacement blower. The blower was gas tight allowing elevated inlet pressures (Model 3204, Tuthill, USA). Seal areas were vented to atmosphere to relieve process pressure against the internal lip seals, and to provide oil-free air. The motor was driven from an inverter (Vacon Drives UK Ltd) and its speed controlled via a 4-20 mA signal from a computer.

As the parallel compressor could not tolerate high inlet temperatures, the air coming from the primary compressor was inter-cooled. This was carried out using water running through a shell and tube heat exchanger (SB4527, EJ Bowman Ltd, UK)

An unfortunate consequence of this inter-cooling was that the air temperature at the exit from the parallel compressor was too low for cooking and high temperature water heating. Therefore this air was fed into the system between the water heater and the hot water pre-heat exchanger, not into the cooking tunnel. 


\section{METHOD}

\subsection{Coefficient of Performance (COP) of Cooling}

During initial experiments it was found that the heat infiltration, thermal mass and pressure drop of the cooling tunnel were higher than would be the case for an optimised system and steady state conditions took prohibitively long to reach.

For these reasons, to measure the COP of cooling, the cooling tunnel was by-passed and different heat loads were created by removing sections of insulation from the cooling pipes (pipes connecting the tunnel to the rest of the apparatus). This allowed COP measurements to be taken at much lower temperatures and heat loads than were possible with the cooling tunnel connected.

To calculate the COP of cooling required measuring the cooling load and electrical power into the compressors:

$$
\mathrm{COP}_{\text {cool }}=\frac{Q_{\text {cool }}}{P}
$$

The power of the primary and parallel compressors were measured using portable energy monitors. The measured power for the compressors was the total power into their control panels and included inverter losses and control circuits. The power for the external supply of compressed air for the primary compressor air bearings was not included.

To calculate the cooling load required measurement of the mass flow rate and temperature difference across the load.

$$
Q_{\text {cool }}=\dot{m} \cdot c_{p} . \Delta T
$$


$\Delta \mathrm{T}$ was taken between the outlet of the turbine and the inlet to the recuperator. The cooling load was therefore through the steel pipes and any insulation. Air temperature after the turbine was measured using a platinum resistance thermometer (PRT) (accuracy $\pm 0.2 \%$ ) inserted through a gland into the centre of the pipe.

A direct method of measuring flow rate was not incorporated in the primary flow pipes as this would have increased pressure losses. Instead, the temperature difference across a length of pipe with a known heat input from an electrical heater tape was used. The heater tape was wrapped around the outside of the pipe downstream of the turbine. The heater tape was well lagged with Armaflex insulation and its temperature kept close to ambient temperature such that the vast majority of the heat went into the pipe and therefore into the air inside the pipe and little escaped into the surroundings. The temperature difference between the air upstream and downstream of the heater tape was measured by placing the cold and hot junction of a thermocouple at these positions, measuring the voltage and converting this to a temperature difference (accuracy $\pm 0.2^{\circ} \mathrm{C}$ ). The thermocouples were placed inside wells welded into the pipes such that their junctions were located at the centre of the pipes.

The system was run for approximately 4 hours until a steady temperature was measured at the outlet of the turbine and a steady temperature difference was measured across the heater tape. The heater tape was then turned on for a further 2 hours until a steady temperature difference was measured across the heater tape. The difference in temperature difference between the conditions with the heater tape on and off was the 
temperature difference caused by the heater tape. These results were used to calculate the mass flow rate as shown below.

$$
\dot{m}=\frac{Q_{h t}}{c_{p} . \Delta T}
$$

A set of experiments was carried out with the thermal insulation intact. This was called the 'low load' condition. At this condition the COP at three different compressor speeds was measured (low speed, medium speed and high speed). The same measurements were made for 'medium' and 'high' load conditions, where increasing amounts of thermal insulation were removed from the cooling pipework. The nine measurement conditions (load and speed) are shown in Table 1.

Mass flow rate through the bootstrap compressor was measured using an orifice plate (M410, Spirax Sarco UK, $\pm 3 \%$ accuracy) and digital pressure transmitter (Model FKK, Fuji Electric Systems Co, Ltd, Japan, $0.1 \%$ accuracy). The orifice plate was located in a section of pipe which bypassed the heating tunnel and was selected to give a similar pressure drop to that of the cooking tunnel. Therefore measurement of bootstrap compressor flow rate was only possible whilst all the flow was diverted around the heating tunnel. This was the case for the COP but not the food measurements.

\subsection{COP of Heating}

Unlike the COP of cooling which is considered to take place at one location (after the turbine), the $\mathrm{COP}$ of heating can be considered at different points around the system and therefore at different temperatures. The $\mathrm{COP}$ of heating was therefore considered at each 
of the three water-to-air heat exchangers in the apparatus. Each of these three heat exchangers serves a different purpose.

1. The hot water heater was designed to exchange heat from the hot air not used in the heating tunnel to heat water in a storage tank to a temperature close to boiling.

2. The hot water pre-heater was designed to reduce the temperature of the air entering the recuperator.

3. The intercooler was designed to reduce the temperature of the air entering the secondary compressor.

For these COP experiments the heating tunnel was bypassed (via the orifice plate) such that air from the outlet of the bootstrap compressor went directly into the hot water heat exchanger.

The COP of heating was calculated for each of the three heat exchangers for the 9 experiments shown in Table 1 using equations 5 and 6.

$$
\begin{aligned}
& \mathrm{COP}_{\text {heat }}=\frac{Q_{\text {heat }}}{P} \\
& Q_{\text {heat }}=\dot{m} \cdot c_{p} \cdot \Delta T
\end{aligned}
$$

The heat losses in the pipework upstream and downstream of the heat exchangers, if significant, were included in the calculation of the heat load, therefore the heat loads in the heat exchangers were in effect the maximum heat available to these heat exchangers.

For the hot water heater, the temperature difference was calculated from the outlet of the bootstrap compressor to the outlet of the hot water heater. For the hot water pre- 
heater, the temperature difference was calculated from the inlet of the hot water pre-heater to the inlet of the recuperator. For the intercooler, the temperature difference was calculated from the outlet of the primary compressor to the outlet of the intercooler.

The mass flow rate of air through the hot water pre-heater was the same as through the cooling section. The mass flow rate of air through the hot water heater was less than through the cooling section as some of the flow went through the parallel compressor. The mass flow rate in this section was calculated by means of the orifice plate. The mass flow rate of air through the intercooler was the mass flow rate through the cooling section minus that through the hot water heater.

\subsection{Efficiencies}

Efficiencies of the rotating machines were estimated using performance maps from the manufacturers. These performance maps are not shown due to confidentiality.

The turbine isentropic efficiency was given as a function of its pressure ratio and velocity ratio. The velocity ratio was a function of the rotating speed and isentropic temperature drop.

The bootstrap compressor isentropic efficiency was given as a function of its pressure ratio and either the aerodynamic speed (equation 7) or a term known as the 'corrected mass flow rate' (equation 8).

$$
\mathrm{v}=\frac{N}{\sqrt{T_{\text {in }}}}
$$




$$
\dot{m}_{c o r}=\frac{\dot{m} \cdot \sqrt{T_{i}}}{p_{i}}
$$

The primary compressor isentropic efficiency was given as a function of its rotating speed and mass flow rate.

Efficiency data for the secondary compressor were not available and therefore isentropic efficiency was calculated using measured inlet and outlet pressures and temperatures.

$$
\eta=\frac{T_{o, i s}-T_{i}}{T_{o}-T_{i}}
$$

Where;

$$
T_{o, i s}=T_{i} \cdot r_{c}^{\frac{\lambda-1}{\lambda}}
$$

\subsection{Food measurements}

For the experiments where food was heated and cooled, the cooling tunnel was not removed and all of the flow from the bootstrap compressor went directly into the heating tunnel before entering the hot water heater. The apparatus was run for 2 hours prior to putting product through the tunnels, to allow the apparatus to get to temperature. The primary compressor was run at $68400 \mathrm{rpm}$.

Product temperatures were measured using specially constructed probes, each consisting of four type $\mathrm{T}$ thermocouples (calibrated at 0 and $100^{\circ} \mathrm{C}$, accuracy $\pm 0.5^{\circ} \mathrm{C}$ ) 
attached to a wooden 'cocktail' stick at $15 \mathrm{~mm}$ intervals. The probes were placed into beef burgers (100\% Beef Quarter Pounders, Birds Eye) radialy such that the four thermocouples spanned from the centre to the edge of the burgers. Probe 4 was at the surface of the burger and probe 1 in the centre. Each beef burger weighed $100 \mathrm{~g}$ and was $90 \mathrm{~mm}$ diameter and $10 \mathrm{~mm}$ thick.

Burgers which had been pre-stabilised to $5^{\circ} \mathrm{C}$, were placed into the heating and cooling tunnels and conveyed through. Temperatures in the burgers were recorded at 5 second intervals during heating and cooling using a data logger (Squirel, Grant Instruments (Cambridge) Ltd).

The inverter frequencies for the tunnel fans were set to $20 \mathrm{~Hz}$ for these experiments. This gave velocities of approximately $6 \mathrm{~m} . \mathrm{s}^{-1}$ out of the impingement holes. Higher inverter frequencies and therefore impingement speeds were possible (35 Hz equated to $11 \mathrm{~m} \cdot \mathrm{s}^{-1}$ ), but these led to a great deal of infiltration between the tunnel and ambient air, thus giving a high ambient load. This was particularly problematic with the cooling tunnel as this meant that more water vapour entered the tunnel causing icing problems.

\section{RESULTS}

\subsection{COP measurements}

Both the compression ratio and mass flow rate of the primary compressor increased with the compressor speed as shown in Figure 3. Maximum pressure ratio of 2.66 and mass flow rate of $0.276 \mathrm{~kg} . \mathrm{s}^{-1}$ were measured at a speed of $66821 \mathrm{rpm}$. 
Primary compressor isentropic efficiencies were between 0.77 and 0.78 . The maximum possible (design) efficiency of the primary compressor was 0.8 . The secondary compressor speed was adjusted with the aim of keeping the primary compressor operating at a high efficiency and that is why the efficiency changes little between tests.

The measured bootstrap compressor isentropic efficiency was between 0.66 and 0.77. The maximum possible (design) efficiency of the bootstrap compressor was 0.83 at a pressure ratio of 1.6 (maximum pressure ratio achieved was 1.3).

The measured turbine isentropic efficiencies were between 0.7 and 0.77 . The maximum possible (design) efficiency of the turbine was 0.81 at a pressure ratio of 3.0 (the highest pressure ratio actually achieved) and 0.84 at a pressure ratio of 4.0 .

The measured parallel compressor isentropic efficiencies were between 0.62 and 0.68 .

The cooling loads used in COP calculations are plotted against the average temperatures that the loads occurred across (Figure 4). As the speed of the primary compressor increased, the temperatures at which the loads occurred decreased. Increasing the cooling loads (by removing insulation) resulted in small increases in the load temperature. There was one exception to this result (medium speed and low load) which was at a lower speed than the other medium speed results (shown in Table 1). If run at the same speed the temperature would be lower, as shown by the arrow in Figure 4. A maximum cooling load of $3.56 \mathrm{~kW}$ was measured at high speed. 
The COPs of cooling are plotted against the average temperatures across which the loads occurred (Figure 5). Increasing the load temperatures and increasing the cooling loads led to increases in COP. A maximum COP of 0.126 was measured at low speed and high load.

The heat loads available to the hot water heater, hot water pre-heater and intercooler are plotted against the average temperatures across which the loads occurred for the maximum speed conditions (Error! Reference source not found.). The overall heat load (sum of heat loads) increases with the cooling load, however, due to valves being adjusted to keep the various parts of the system working within design conditions, different heat exchangers take a different proportion of the heat load between tests. This is why the heat load at each heat exchanger does not always increase significantly with cooling load.

The hot water heater generated the least heat $(10 \mathrm{~kW})$ at the highest temperature 140 to $150^{\circ} \mathrm{C}$. The intercooler generated the highest heat $(18$ to $19 \mathrm{~kW})$ at just under $100^{\circ} \mathrm{C}$. The hot water pre-heater generated its heat $(12$ to $14 \mathrm{~kW}$ ) at the lowest temperature (62 to $65^{\circ} \mathrm{C}$ ).

The COPs for the different heat exchangers (heating and cooling) are plotted against the temperatures across which the loads occurred for the high speed high load conditions (Figure 7). The highest COP (0.37) was for the intercooler with inlet and outlet temperatures of $153^{\circ} \mathrm{C}$ and $46^{\circ} \mathrm{C}$ respectively.

An analysis of the accuracy of the presented COP and heating/cooling load derived from the instrumentation accuracy for the high speed, high load condition has been carried out and is shown in Table 2. 


\subsection{Food tests}

Within 20 minutes of chilled burgers entering the cooling tunnel, their centre temperatures were approximately $-19^{\circ} \mathrm{C}$ and their surface temperatures approximately $-44^{\circ} \mathrm{C}$ (Figure 8$)$.

Within 9 minutes of the chilled burgers entering the heating tunnel their centre temperatures were approximately $75^{\circ} \mathrm{C}$ (Figure 9).

\section{CONCLUSIONS}

A closed air cycle cooling and heating apparatus has been built and tested for use in the food industry. The apparatus used a bootstrap unit developed for aircraft air conditioning in a system modified to run at the low temperature required for food freezing, by using a parallel compressor. This approach allowed temperatures as low as $-140^{\circ} \mathrm{C}$ leaving the turbine and as high as $234^{\circ} \mathrm{C}$ leaving the bootstrap compressor.

Large quantities of heat which could be used for heating water, steam or cooking food were produced when generating cold temperatures. The system was able to rapidly cook and freeze beef burgers using air impingement, and at the same time heated water at temperatures up to almost boiling point.

With the air cycle system operating at an average cooling heat exchanger temperature of $-100^{\circ} \mathrm{C}$, with a COP of 0.085 and an electricity cost of $£ 0.05$ per $\mathrm{kWh}$, the cost per kWh of cooling is $£ 0.69$. A comparable LIN system would use $12.2 \mathrm{~kg}$ of LIN, which at $£ 0.06$ per $\mathrm{kg}$, would cost $£ 0.73$. In this analysis the air cycle is cheaper to run than a LIN system with the added benefit of a large amount of free heating. 
The use of bootstrap units designed for the purpose of cooking and freezing food would allow the removal of the parallel compressor making the system more simple and efficient. The use of heating tunnels designed for use with air cycle would also allow more efficient freezing and cooking of food.

This system has been shown to produce cooling at cryogenic temperatures, with waste heat capable of cooking and boiling water. For these reasons it offers a viable alternative to current systems used in food factories and the other systems described in the introduction.

\section{ACKNOWLEDGEMENTS}

The authors wish to thank the UK Department for Environment, Food and Rural Affairs (Defra) for funding this project under the Advanced Food Manufacturing Link scheme. The authors also wish to thank the project partners Air Products, Avana Bakeries

Ltd, Campden BRI, Chris Jessop Energy Consulting, Earthcare Products, Bakkavör, Hygrade Foods Ltd, Masterfoods and Munters. 


\section{REFERENCES}

Anon. Frozen Foods UK - Market Report 2009, 24th Edition, Keynote, ISBN 978-1-84729$513-2$

Benois, H.G., 1974. Freezing with air at minus $80^{\circ}$ F. Agricultural Research Council Symposium No. 3, Meat Freezing - Why and How? April 1974, MRI, Langford, Bristol.

Das, F., 2000. Integrated heating and cooling in the food and beverage industry, Food and Beverage Industry, Newsletter No. 2.

Elsayed ES, Akisawa A, Kashiwagi T, Hamamoto Y. 2006. Using air cycle refrigerator integrated desiccant system for simultaneous usage in supermarket: refrigerating and ventilated air conditioning. Proc. of the 3rd Asian Conference on Refrigeration and AirConditioning Vols I and II (ACRA2006). May 21-23, 2006, Gyeongju, Korea

Giles, G.R., 1998. Environmentally friendly air cycle air conditioning. Proceedings of the Institute of Refrigeration, 1999-9. 1-1.

Hou S, Zhang H. 2009, An open reversed Brayton cycle with regeneration using moist air for deep freeze cooled by circulating water. International Journal of Thermal Sciences 48(1): 218-223.

Hou SB, Li HC, Zhang HF. 2008, An open air-vapor compression refrigeration system for air-conditioning and desalination on ship. Desalination 222(1-3): 646-655. 
Kauffeld, M., König, H., Kruse, H., 1991. Theoretical and experimental evaluation of the potential of air cycle refrigeration and air conditioning. In Proceedings of 18th International Congress on Refrigeration, Montreal, 1991, pp. 1124-1128.

Pearson, S.F., 2006. Highly efficient water heating system. IIR 7th Gustav Lorentzen Conference on Natural Working Fluids, Trondheim, Norway, 2006.

Regmi, A., and Gehlhar, M., 2005. New Directions in Global Food Markets. Agriculture Information Bulletin No. 794, p 81.

Rogers, B.H., 1994. Cooling in aircraft. Proceedings of the Institute of Refrigeration, 199495,4-1.

Sarkar, J., Bhattacharyaa, S. and Gopal, M.R., 2006. Simulation of transcritical CO2 heat pump for simultaneous cooling and heating applications. International Journal of Refrigeration, 29,735-743.

Spence SWT, Doran WJ, Artt DW and McCullough G. 2005, Performance analysis of a feasible air-cycle refrigeration system for road transport. International Journal of Refrigeration-Revue Internationale Du Froid 28(3): 381-388. 


\section{FIGURES}

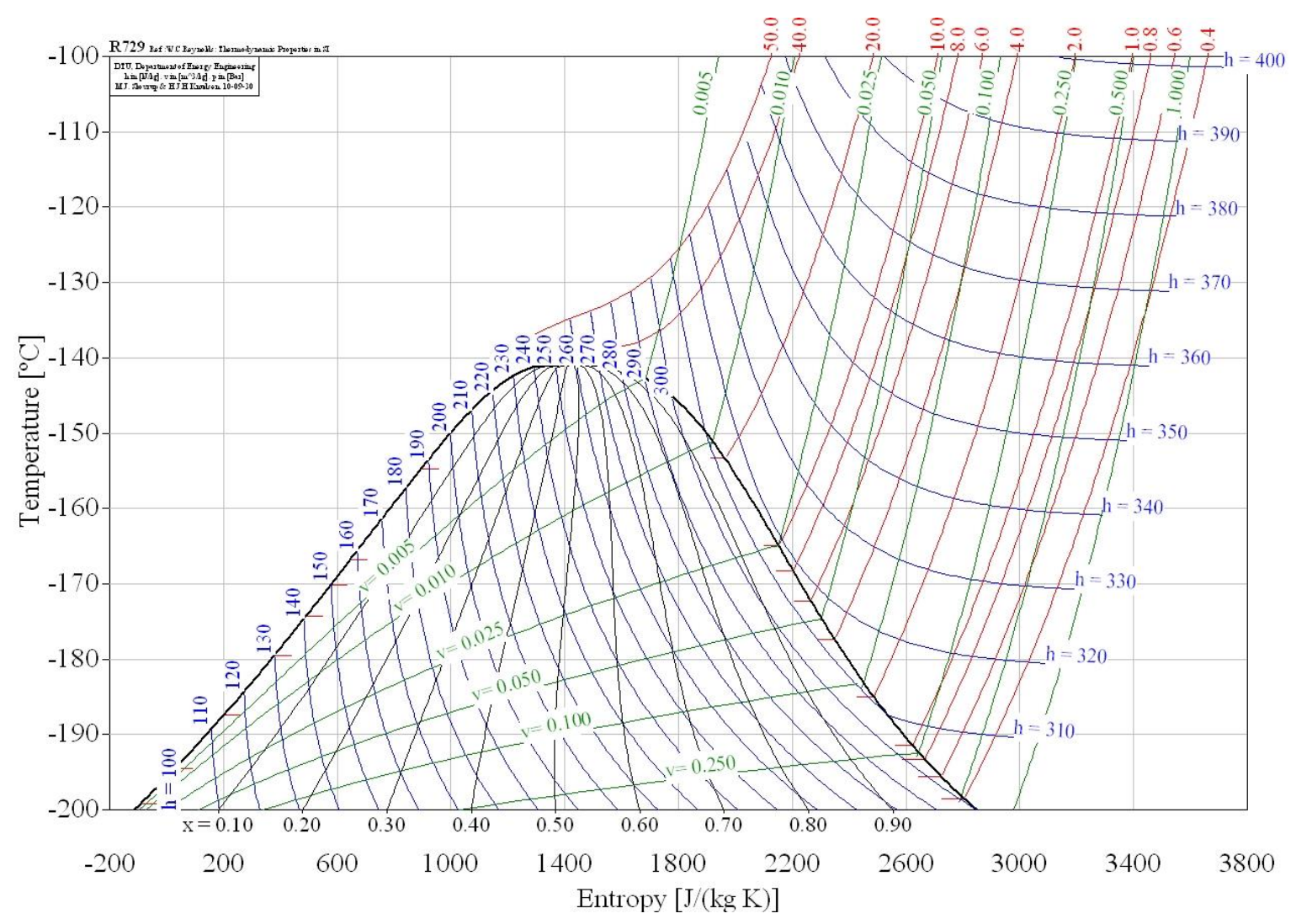

Figure 1. T-s diagram of the air cycle 


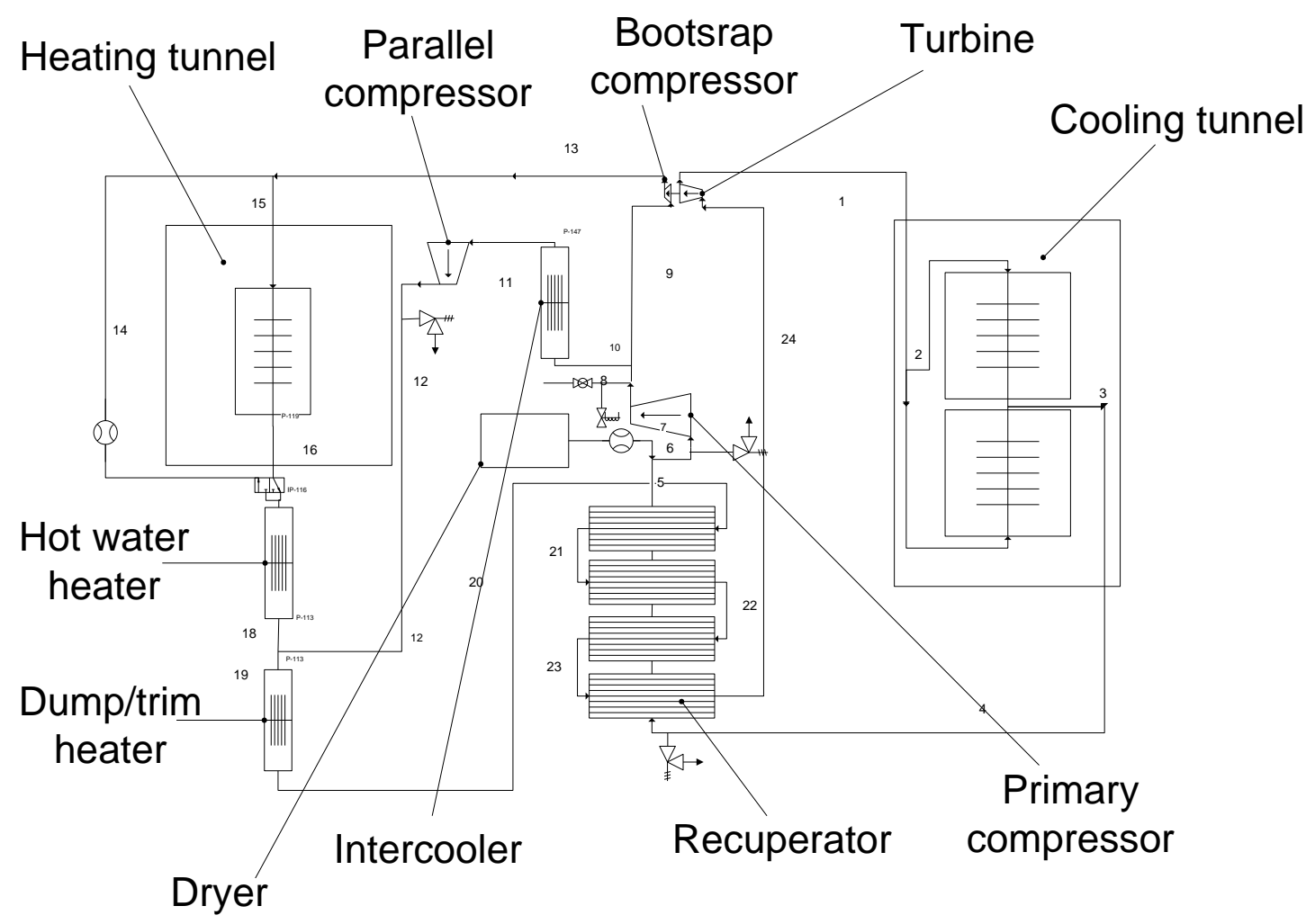

Figure 2. Schematic of the equipment 


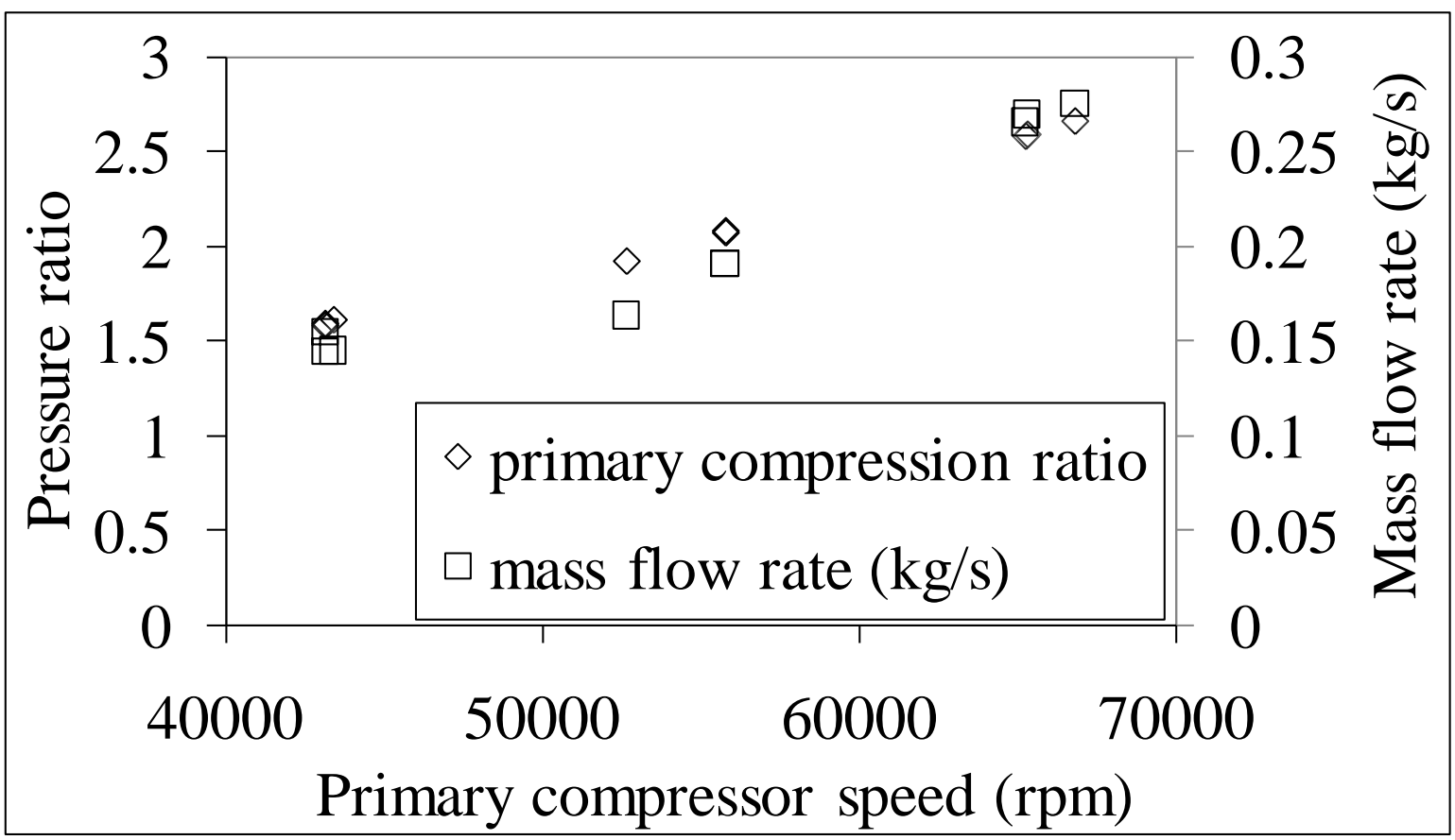

Figure 3. Compression ratio and mass flow rate of the primary compressor for given rotational speeds. 


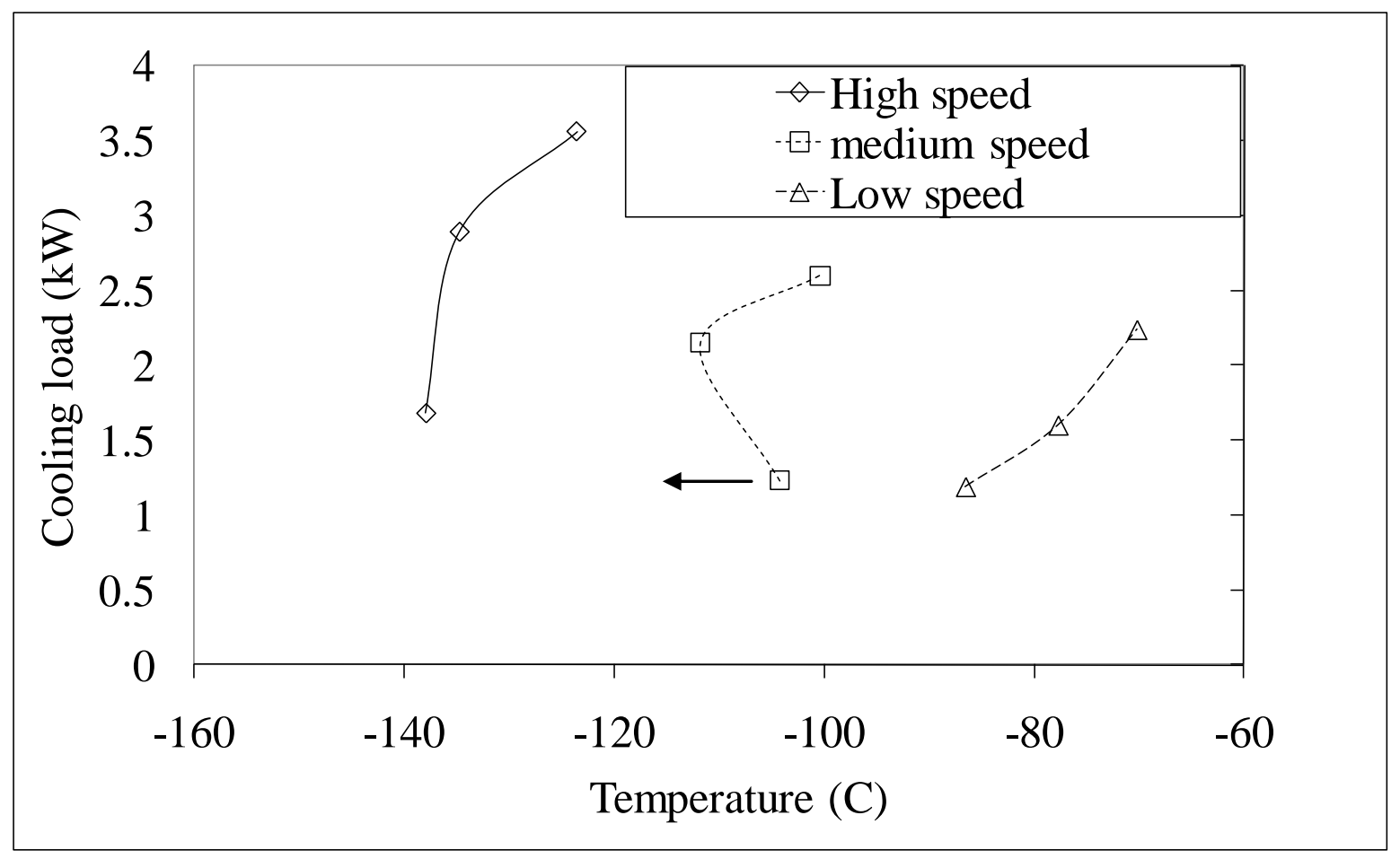

Figure 4. Cooling load is plotted against the average temperature across the load. 


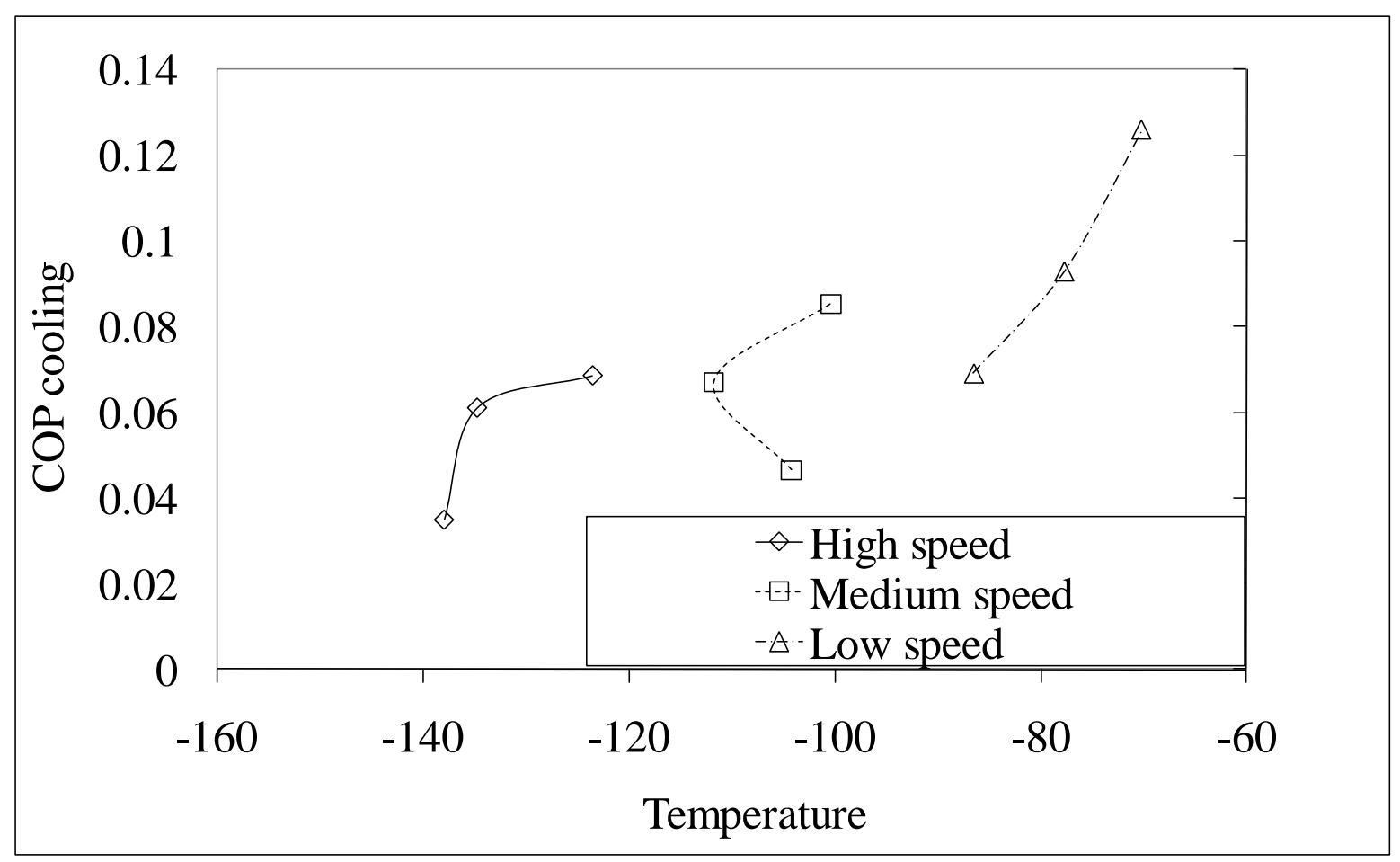

Figure 5. COP of cooling is plotted against the average temperature across the load. 


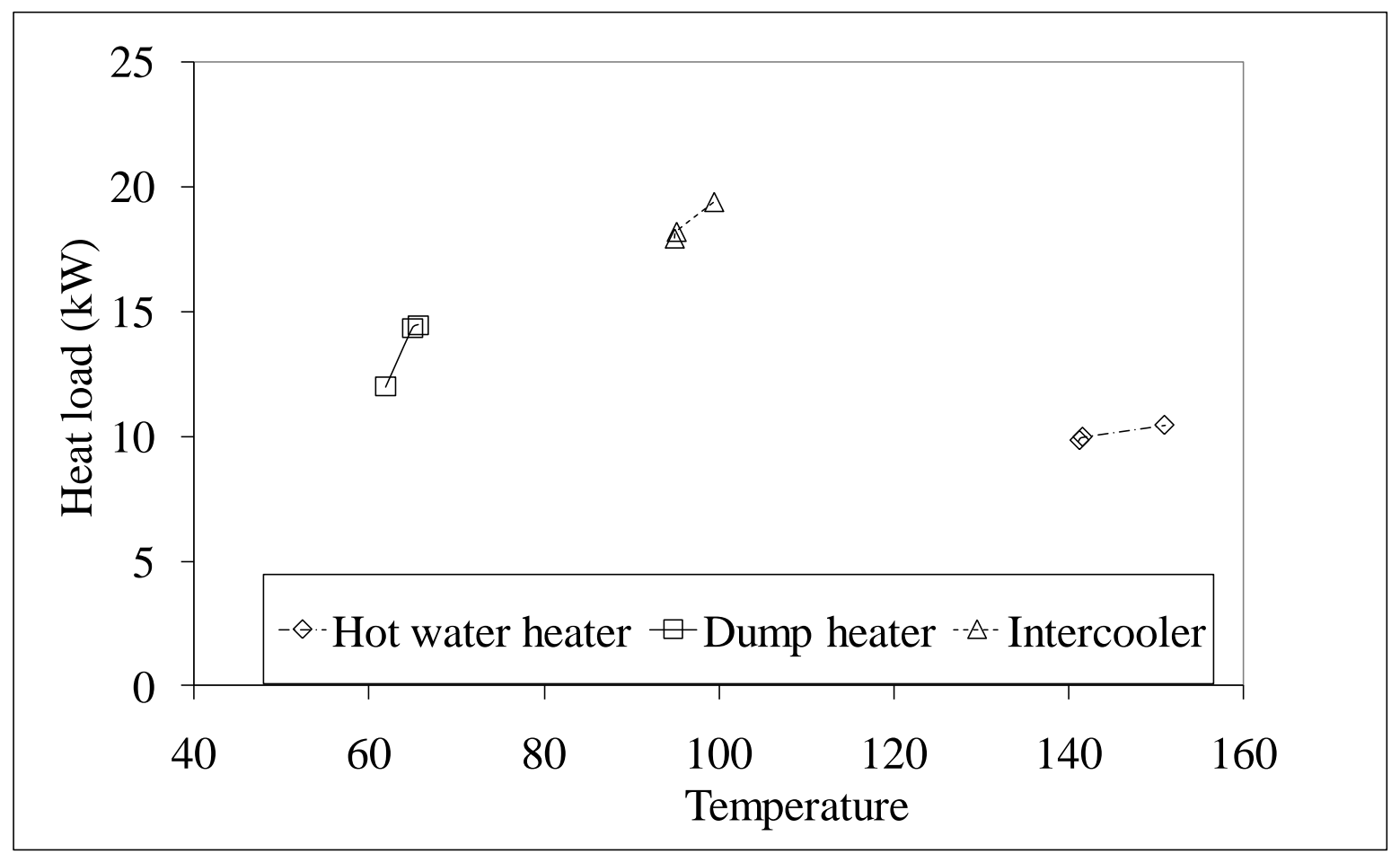

Figure 6. The heat load available to the hot water heater, hot water pre-heater and intercooler against the average temperature across the load for the maximum speed condition. 


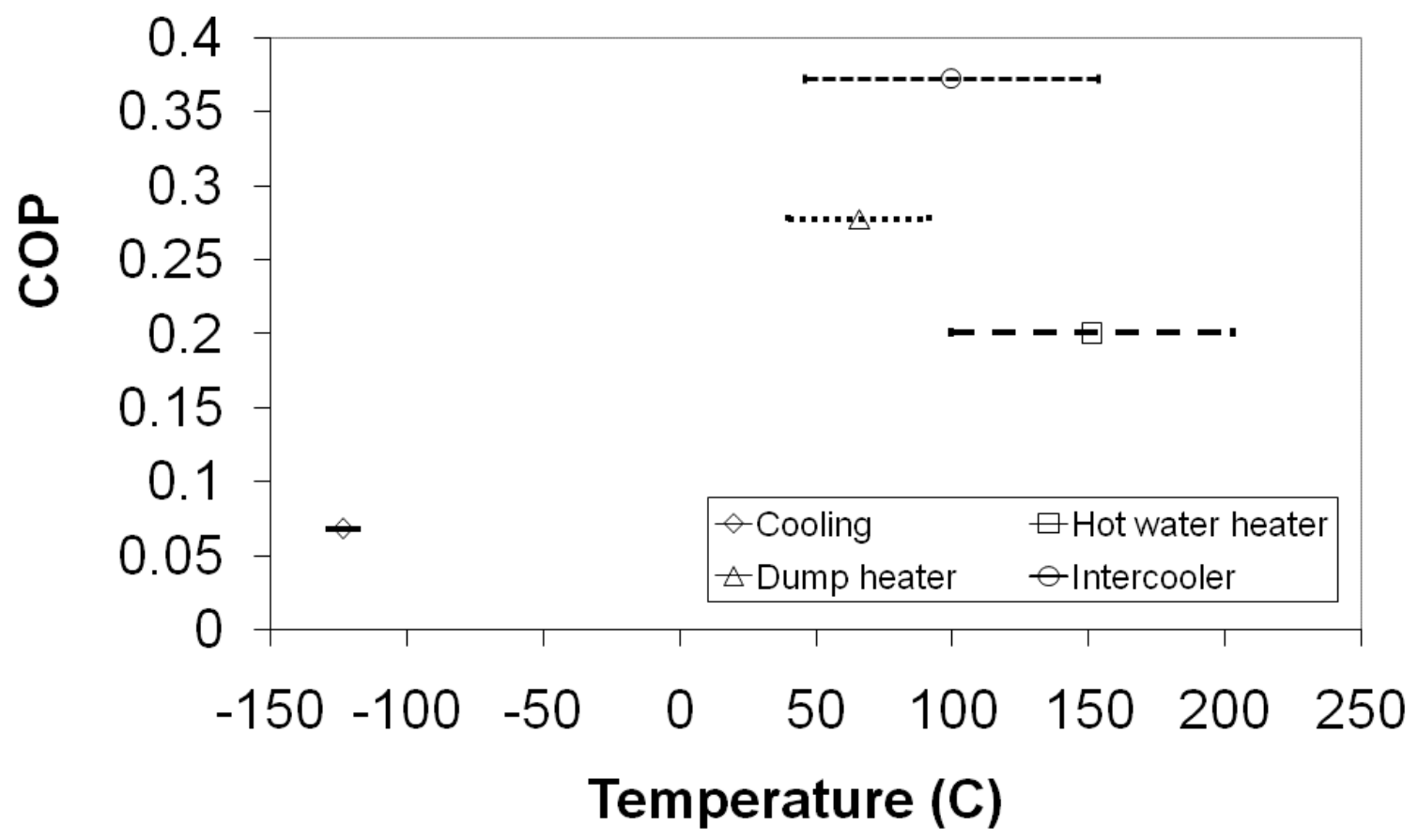

Figure 7. The COP for the different heat exchangers against the average temperature across the load for the maximum speed, maximum load condition. 


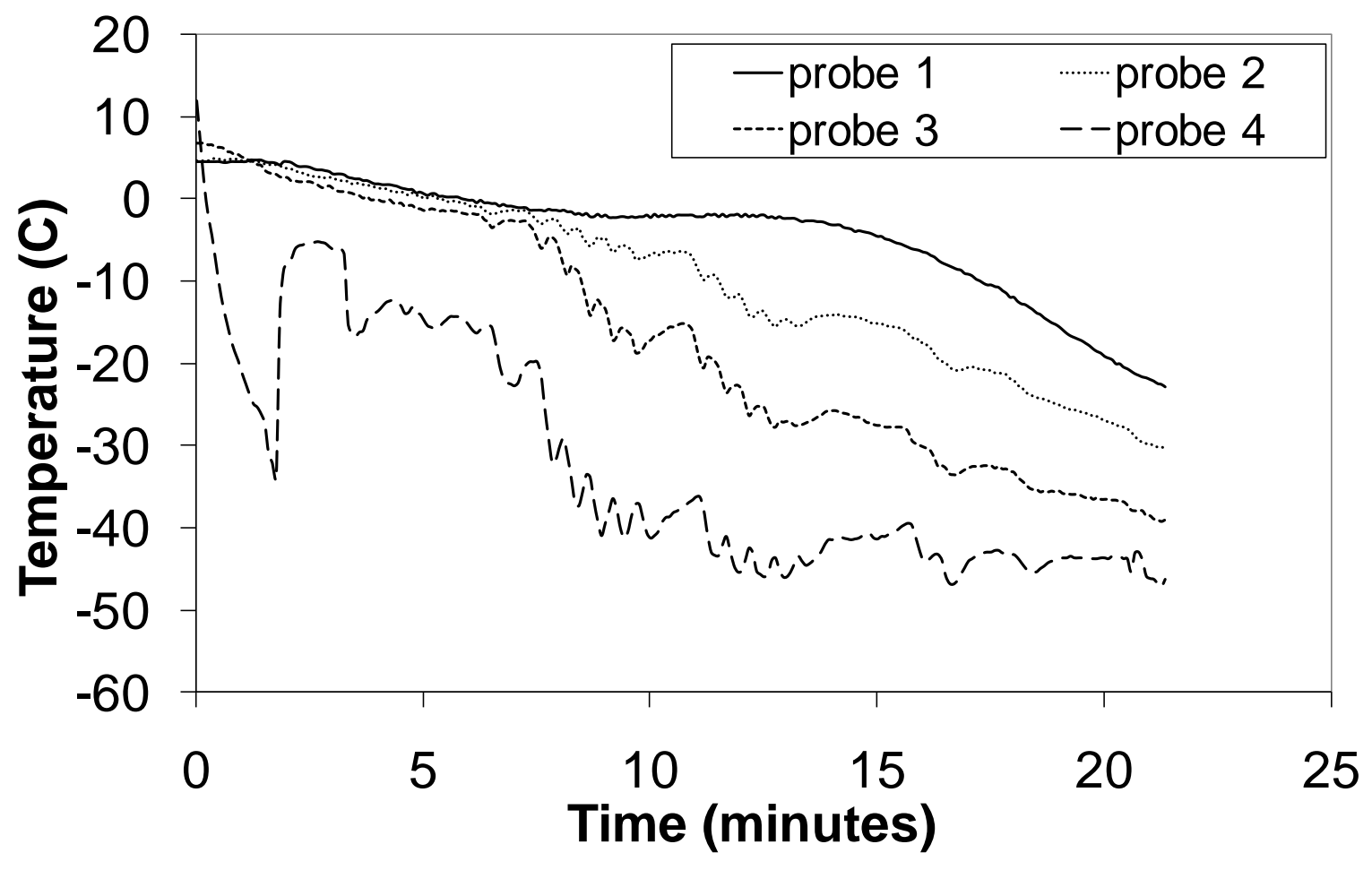

Figure 8. Beef burgers frozen in cooling tunnel. 


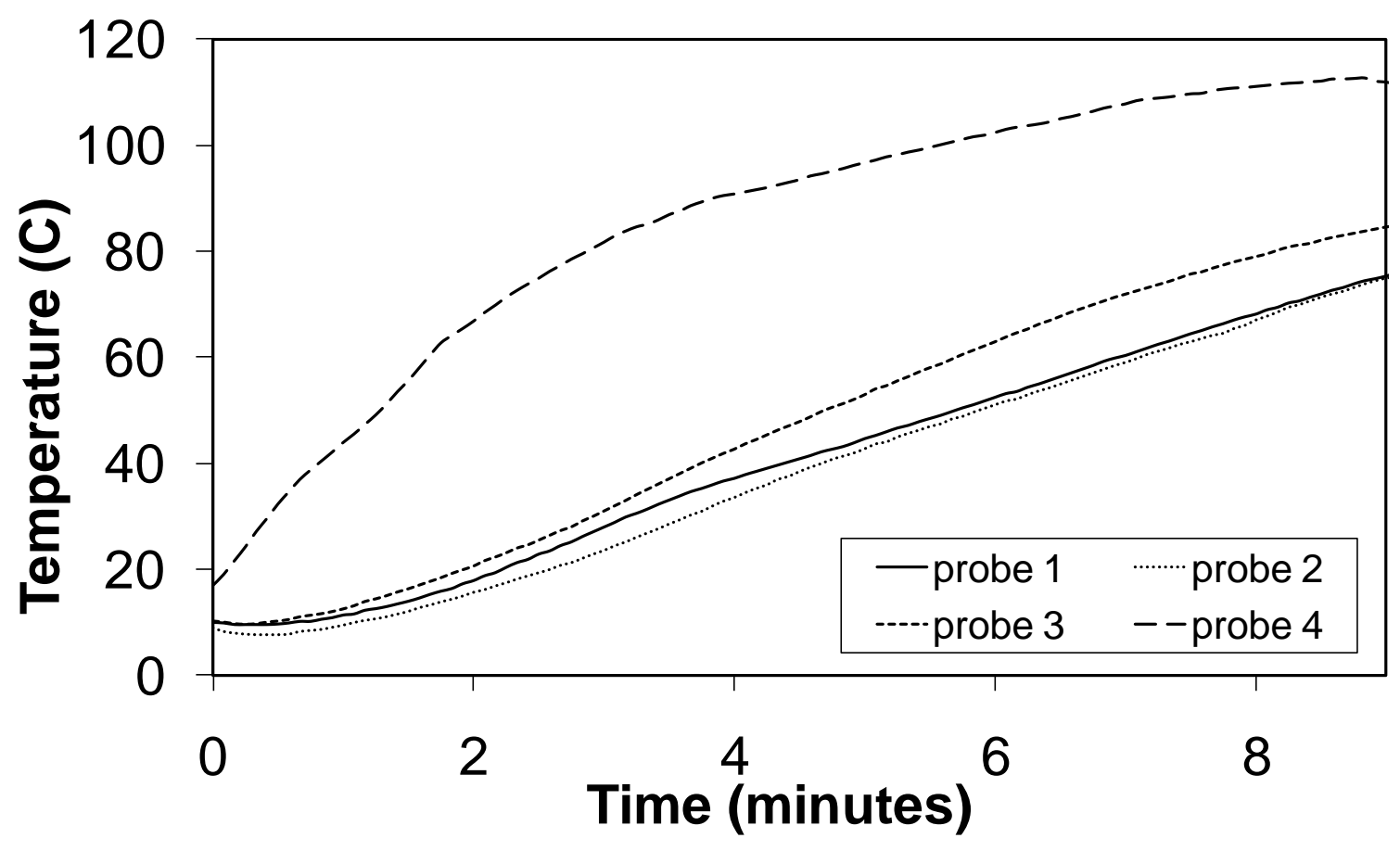

Figure 9. Beef burgers cooked in heating tunnel 


\section{Tables}

Table 1. Conditions used in COP experiments.

\begin{tabular}{|l|l|l|}
\hline Load & Speed & $\begin{array}{l}\text { Primary compressor speed } \\
\text { (rpm) }\end{array}$ \\
\hline low & low & 43414 \\
low & Medium & 52662 \\
medium & high & 65265 \\
medium & low & 43144 \\
medium & Medium & 55790 \\
high & high & 65320 \\
high & low & 43144 \\
high & Medium & 55788 \\
food & high & 66821 \\
\hline
\end{tabular}


Table 2. Accuracy of the presented COP and heating/cooling load derived from the instrumentation accuracy for the high-speed high-load condition.

\begin{tabular}{|c|c|c|}
\hline Variable & Measurement/calculation & Accuracy $( \pm \%)$ \\
\hline$Q_{h t}$ & e-Tracker Mk2A, Sinergy Ltd. & 4 \\
\hline$\Delta T_{h t}$ & Thermocouple & 2 \\
\hline$\dot{m}_{\text {cool }}$ & $\dot{m}_{\text {cool }}=\frac{Q_{h t}}{c_{p} . \Delta T_{h t}}$ & 6 \\
\hline$\Delta T_{\text {cool }}$ & Thermocouples & 4 \\
\hline$Q_{\text {cool }}$ & $Q_{\text {cool }}=\dot{m}_{\text {cool }} \cdot c_{p} \cdot \Delta T_{\text {cool }}$ & 10 \\
\hline$P$ & e-Tracker Mk2A, Sinergy Ltd. & 2 \\
\hline$C O P_{\text {cool }}$ & $\mathrm{COP}_{\mathrm{cool}}=\frac{Q_{\text {cool }}}{P}$ & 12 \\
\hline$\Delta T_{\text {intercooler }}$ & Thermocouples & 0.3 \\
\hline$\Delta T_{\text {hot water heater }}$ & Thermocouples & 1 \\
\hline$\Delta T_{\text {dump heater }}$ & Thermocouples & 0.2 \\
\hline$\dot{m}_{\text {intercooler }}$ & $\begin{array}{l}\text { Turbine flow meter, Gems } \\
\text { sensors }\end{array}$ & 3 \\
\hline$\dot{m}_{\text {hot water heater }}$ & $\begin{array}{c}\text { Turbine flow meter, Gems } \\
\text { sensors }\end{array}$ & 3 \\
\hline$\dot{m}_{\text {dump heater }}$ & $\begin{array}{c}\text { Turbine flow meter, Gems } \\
\text { sensors }\end{array}$ & 5 \\
\hline$Q_{\text {intercooler }}$ & $Q_{\text {intercooler }}=\dot{m} \cdot c_{p} \cdot \Delta T_{\text {intercooler }}$ & 3 \\
\hline$Q_{\text {hot water heater }}$ & $Q_{\text {hotwaterhater }}=\dot{m} \cdot c_{p} \cdot \Delta T_{\text {hotwaterhater }}$ & 4 \\
\hline
\end{tabular}




\begin{tabular}{|c|c|c|}
$Q_{\text {dump heater }}$ & $\begin{array}{c}Q_{\text {dumpheater }}=\dot{m} \cdot c_{p} \cdot \Delta T_{\text {dumpheater }} \\
C O P_{\text {intercooler }}\end{array}$ \\
$\mathrm{COP}_{\text {intercoole }}=\frac{Q_{\text {intercooler }}}{P}$ & 5 \\
$C O P_{\text {hot water heater }}$ & $\mathrm{COP}_{\text {hot waterheater }}=\frac{Q_{\text {hotwaterheter }}}{P}$ \\
$\mathrm{COP}_{\text {dump heater }}$ & $\mathrm{COP}_{\text {dumpheater }}=\frac{Q_{\text {dumpheater }}}{P}$ & 6 \\
& & 7
\end{tabular}

